\title{
Geschichte der Tumeszenz-Lokalanästhesie ${ }^{1}$
}

\author{
History of Tumescent Local Anesthesia
}

Autor

Institut
H. Breuninger

Universitäts-Hautklinik, Eberhard-Karls-Universität, Tübingen

\section{Bibliografie}

DOI 10.1055/s-0029-1214739

Online-Publikation: 20. 5. 2009

Akt Dermatol 2009; 35 :

290-292 @ Georg Thieme

Verlag KG Stuttgart · New York

ISSN 0340-2541

Korrespondenzadresse

Prof. Dr. Helmut Breuninger

Universitäts-Hautklinik Eberhard-Karls-Universität Liebermeisterstraße 25 72076 Tübingen

Helmut.Breuninger@

med.uni-tuebingen.de

\section{Zusammenfassung \\ $\nabla$}

Die Tumeszenz-Lokalanästhesie bedeutet eine wirksame Anästhesie mit bis zu 100-fach verdünnten Lösungen der üblichen Lokalanästhetika. Dadurch ist es möglich, auch die 100-fache Menge zu applizieren. Dies erweitert die Anwendungsmöglichkeiten der Lokalanästhesie. Dass diese Verdünnung mit Cocain wirksam ist, hatte schon der Chirurg Schleich im 19. Jahrhundert 1892 berichtet. Damals ist die Idee wegen der aufkommenden Äthernarkose nicht weiter verfolgt worden. Erst J. Klein hat diese Idee im Rahmen der Liposuktion 1987 neu erfunden. Er verwandte dabei Lidocain, das 1942 auf den Markt kam. G. Sattler führte sie in Deutschland 1994 ein, ebenfalls im Rahmen der Liposuktion. Er benutzte früh eine Rollenpumpe zur Applikation. Kontrovers wurde die maximal zu applizie-

\section{Einleitung}

\section{$\nabla$}

Lange war die Lokalanästhesie auf kleinere Areale begrenzt, da bei den verwendeten Konzentrationen von 2 bis $0,5 \%$ die bisher geltende Höchstdosis rasch erreicht war. Eine vollkommen neue Situation wurde erreicht, als Jeffry Klein im Rahmen der Anwendung bei der Liposuktion feststellte, dass auch hundertfach verdünnte Lösungen anästhetisch wirken [1]. Dadurch war es möglich geworden, auch die 100-fach größere Menge als bisher zu applizieren. Durch die großzügige Volumengabe kommt es zum Anschwellen der infiltrierten Subkutis, was so viel wie „tumeszere“ bedeutet. Deshalb wurde der Namen „Tumeszenz-Lokalanästhesie“ (TLA) kreiert.

Genau diese Tatsache, dass eine starke Verdünnung von Lokalanästhetika wirksam ist, hatte

1 45. DDG-Tagung 29.4.-2.5.2009 Dresden, Arbeitsgemeinschaft Geschichte der Dermatologie (AGDV) rende Menge diskutiert. Schon bald wurde klar, dass die Möglichkeiten dieser Technik nicht auf diese Indikation beschränkt waren. Venenstripping und viele Hautoperationen wurden damit durchgeführt. H. Breuninger entwickelte 1998 die subkutane Infusionsanästhesie (SIA), eine automatisierte langsame TLA mit Flow- und Volumen-kontrollierten Infusionsautomaten (AutoTLA). Zudem führte er 1999 das lang wirksame Lokalanästhetikum Ropivacain in der Mischung ein. Durch die lang anhaltende Anästhesie konnte die Lokalanästhesie vom Eingriff zeitlich getrennt werden. Damit erweiterte sich die Indikation der TLA auf nahezu alle Eingriffe an der Haut. Auch für Kinder war die Methode nun zugänglich. 2006 wies H. M. Häfner nach, dass die adrenalinhaltige Lösung auch unbedenklich an den Akren verwendet werden kann. Die Entwicklung der TLA ist also noch nicht abgeschlossen.

schon der Chirurg Schleich im 19. Jahrhundert 1892 berichtet.

In den Verhandlungen der deutschen Gesellschaft für Chirurgie (Verh. Dtsch. Ges. Chir.) vom 11. Juni 1892 [2] wird Folgendes schriftlich belegt (๑ Abb. 1):

Herr Schleich aus Berlin hält einen Vortrag über die Infiltrationsanästhesie (Lokale Anästhesie) und ihr Verhältnis zur allgemeinen Narkose (Inhalationsanästhesie).

Er hat festgestellt, dass Verdünnungen des damals zur Verfügung stehenden Cocains von 1 zu 5000 noch genauso anästhetisch wirksam waren. Er fand weiter heraus, dass eine 0,2\%ige Kochsalzlösung anästhetisch wirksam ist. Nun hat er beide Erfahrungen kombiniert und in der Praxis angewandt. Er hat dies an 512 Patienten erfolgreich getestet. Seine Operationen waren Gastrostomie, Herinotomie, Mammaablatio, regionäre Dissektionen, Nierennähte, Sequestrotomiene und Amputationen. Zwischenfälle traten nicht auf. Er hat 
Einundzwanzigster Congress. 4. Sitzungstag, 11. Juni 1892. 121

2) Herr Sohleioh (Berlin): Die „Infiltrationsanästhesie (lo oale Anästhesie) and ihr Verhältniss zur allgemeinen Narcose (Inhalationsanästhesie)." M. H.! Die bisherigen Bestrebungen, Opera-

M. H.! Eine Methode der Anästhesie, welche es gestattet hat, Cysten von 10 Liter Inhalt schmerzlos ans der Banchhöhlo ohne Spur von Chloroform zu entfernen - auch solohe Fälle habe ich in der hiesigen medioinischen Gesellschaft vorzustellen die Ehre gehabt, ebenso wie einen Fall von Gastrostomi - eine Methode, welohe ohne jede Chloroformanwendung sohmerzlos Nierennähte, Herniotomion, Sequestrotomien, Mamma-Amputationen mit Ansräumung der Achsel und den Bauchsohnitt auszuführen orlaubte - während bei der an 521 Kranken erprobten Technik auch kein Sohatten einer Gefabr sich herausstellte - eine Methode, bei welchor eine Intoxioationsgefahr geradoza zur Unmöglichkeit geworden ist, eine solche wird meiner Meinung nach ganz von selbst in die Schranken treten mit der immorhin gefährliohen, allgemeinen Narcose, mit all' ihren Schattenseiten, unangenehmen und krankmachenden Nebenumständen. Denn darüber darf dooh nioht binweggesehen
Abb. 1 Auszug aus der Orginalpublikation über die Rede von C. L. Schleich vor der Gesellschaft für Chirurgie am 11.6. 1982. dann erklärt, „dass man bei allen Operationen, die in dieser Art der Lokalanästhesie möglich wären, auf die Inhalationsanästhesie verzichten soll, da sie eine höheres Risiko für den Patienten habe. Ja er meinte, dass der Arzt moralisch verantwortlich wäre, das risikolosere Verfahren der Anästhesie zu wählen und sich eventuell strafbar machen würde, wenn in solch einem Fall der unnötigen Narkose der Patient zu Schaden käme“. Nun hat die versammelte Chirurgenschaft ihm diesen letzten Satz so übel genommen, dass er ab sofort mit seiner Methode tot geschwiegen wurde.

Damals ist also die Idee der Wirksamkeit verdünnter Lokalanästhesielösungen wegen der Eitelkeiten der Verfechter der Inhalationsnarkose unterdrückt worden. Erst nahezu hundert Jahre später hat, wie schon erwähnt, Jeffry Klein diese Idee im Rahmen der Liposuktion 1987 neu erfunden. Gleichzeitig hat er nachgewiesen, dass Lidocain, das 1942 auf den Markt gekommen war, viel besser vertragen wird, als bisher angenommen. Bei subkutaner Verabreichung waren dreifach höhere Dosierungen (35 mg/ $\mathrm{kg}$ Körpergewicht) noch tolerabel [3]. Dennoch wurde die maximal zu applizierende Menge kontrovers diskutiert und Todesfälle auf sie zurückgeführt [4]. Dieses Argument konnte allerdings entkräftet werden. Gerhard Sattler führte die Tumeszenz-Lokalanästhesie in Deutschland 1994 ein [5,6], ebenfalls im Rahmen der Liposuktion. Er benutzte früh eine Rollenpumpe zur Applikation. Schon bald wurde klar, dass die Möglichkeiten dieser Technik nicht auf diese Indikation beschränkt waren. Venenstripping und viele Hautoperationen wurden damit durchgeführt (৫ Tab. 1).

Helmut Breuninger entwickelte 1998 die subkutane Infusionsanästhesie (SIA), eine automatisierte langsame TLA mit Flow- und Volumen-kontrollierten Infusionsautomaten (Auto-TLA) [7]. Damit erweiterte sich die Indikation der TLA auf nahezu alle Eingriffe an der Haut. Zudem führte er 1999 das lang wirksame und sehr gut verträgliche Lokalanästhetikum Ropivacain, das 1994 auf den Markt kam, in der Mischung ein [8]. Durch die lang anhaltende Anästhesie konnte die Lokalanästhesie vom Eingriff zeitlich getrennt werden und die lange Schmerzausschaltung war für den Patienten noch komfortabler. Durch die Halbierung der Äquivalenzdosis beider Lösungen halbierten sich auch deren Nebenwirkungen. Auch für Kinder war die Methode nun zugänglich [9].

\begin{tabular}{|c|c|}
\hline 1892 & Erstbeschreibung: Schleich C. L. (Verh Dtsch Ges Chir) \\
\hline 1987 & Wiederentdeckung: Klein J. A. (Am J Cosmet Surg) \\
\hline 1990 & $\begin{array}{l}\text { Hohe Dosen von Lokalanästhetikum sind möglich: J. A. Klein } \\
\text { (J Dermatol Surg Oncol) }\end{array}$ \\
\hline 1992 & $\begin{array}{l}\text { Chirurgie der Kopfhaut: Coleman W. P., Klein J. A. } \\
\text { (J Dermatol Surg Oncol) }\end{array}$ \\
\hline 1995 & $\begin{array}{l}\text { Venenchirurgie: Cohn M. S., Seiger E., Goldmann S. } \\
\text { (J Dermatol Surg) }\end{array}$ \\
\hline 1998 & $\begin{array}{l}\text { Wiedereinführung in Deutschland: Sattler G., Rapprich S., } \\
\text { Hagedorn M. }(\mathrm{H}+\mathrm{G})\end{array}$ \\
\hline 1998 & $\begin{array}{l}\text { Subkutane Infusionsanästhesie: Breuninger H., Wehner-Caroli } \\
\text { J. (J Dermatol Surg) }\end{array}$ \\
\hline 1999 & $\begin{array}{l}\text { Ropivacain in der Tumeszenzlokalanästhesie: Breuninger H., } \\
\text { Hobbach P. S., Schimek F. (J Dermatol Surg) }\end{array}$ \\
\hline 1999 & $\begin{array}{l}\text { Todesfälle nach Liposuktion: Rao R. B., Ely S. F., Hoffmann R. S. } \\
\text { (New Engl J Med) }\end{array}$ \\
\hline 2005 & $\begin{array}{l}\text { Adrenalin an Akren ist ohne Probleme möglich: Häfner H. M. } \\
\text { (JDDG) }\end{array}$ \\
\hline
\end{tabular}

2006 wies Hans Martin Häfner nach, dass die adrenalinhaltige Lösung auch unbedenklich an den Akren verwendet werden kann $[10,11]$. Die Entwicklung der TLA ist also noch nicht abgeschlossen.

\section{Abstract}

\section{History of Tumescent Local Anesthesia}

$\nabla$

Tumescent local anesthesia (TLA) means an effective anesthesia with about 100 fold lower concentrated solutions than usual. Therefore it is possible to apply 100 fold more volume of the solutions. This extended the indications for the use of local anesthesia. In 1892, C. L. Schleich had experimented successfully with this principle of dilutions using Cocaine and performed huge operations in local anesthesia. But this good idea was not further supported because of the more and more used inhalation anesthesia. About 90 years later in 1987, J. Klein again had this idea in the use of local anesthesia for liposuction using Lidocaine, which was in- 
troduced in 1942. G. Sattler introduced the method again in Germany in 1994. He used a roller pump for injection. A controverse discussion took place about the maximal doses which could be applied without danger.

Soon a lot of new application fields for operations in local anesthesia were created, such as vein stripping and large skin-operations. In 1998, H. Breuninger developed the subcutaneous infusion anesthesia with flow and volume controlled infusion automats (auto-TLA). In 1999, he combined the solution with the new long-lasting and very safe local anesthetic ropivacaine which was introduced in 1994. The long lasting anesthesia allowed the division of the application and operation. Nearly all surgical procedures could be performed in local anesthesia, even in children.

In 2006, H. M. Haefner showed that epinephrine can be used without danger at all acres. The development of TLA is not yet finished.

\section{Literatur}

1 Klein JA. The tumescent technique for liposuction surgery. Am J Cosmet Surg 1987; 4: 263-267

2 Schleich CL. Die Infiltrationsanästhesie (lokale Anästhesie und ihr Verhältnis zur allgemeinen Narkose (Inhalationsanästhesie). Verh dtsch Ges Chir 1892; 1: 121

3 Klein JA. Tumescent technique for regional anesthesia permits lidocaine doses of $35 \mathrm{mg} / \mathrm{kg}$ for liposuction: peak plasma lidocaine levels are diminished and delayed 12 hours. J Dermatol Surg Oncol 1990; 16: $248-263$

4 Rao RB, Ely SF, Hoffmann RS. Deaths related to liposuction. New Engl J Med 1999; 13: $1471-1475$

5 Sattler G, Rapprich S, Hagedorn M. Tumeszenzanästhesie - Untersuchungen zur Pharmakokinetik von Prilocain. H + G 1998; 7: 522-525

6 Sommer B, Sattler G, Hankke CW. Die Tumeszenz-Lokalanästhesie. Heidelberg: Springer, 1999

7 Breuninger $\mathrm{H}$, Wehner-Caroli J. Slow Infusion Tumescent Anesthesia (SITA). J Dermatol Surg 1998; 24: 759-763

8 Breuninger H, Hobbach PS, Schimek F. Ropivacaine: An important anesthetic agent for slow infusion and other forms of tumescent anesthesia. J Dermatol Surg 1999; 25: 799-802

9 Möhrle M, Breuninger $H$. Dermatosurgery of children of subcutaneous infusion anesthesia (SIA) with prilocaine and ropivacaine. Pediatric Dermatology 2001; 18: 469-472

10 Häfner HM, Röcken M, Breuninger H. Epinephrine-supplemented local anesthetics for ear and nose surgery: Clinical use without complications in more than 10000 surgical procedures. JDDG 2005; 3: $195-$ 199

11 Breuninger $H$. Dermopix und die Hautchirurgie. Heidelberg: Steinkopff/Springer, 2008 